\title{
Prospective Experiment for Reinforcement Learning on Demand Response in a Social Game Framework
}

\author{
Lucas Spangher ${ }^{*}$ \\ lucas_spangher@berkeley.edu \\ University of California at Berkeley, \\ CA 94709 \\ Joseph Palakapilly \\ jjp126@berkeley.edu \\ University of California at Berkeley, \\ CA 94709
}

\author{
Akash Gokul \\ akashgokul@berkeley.edu \\ University of California at Berkeley, \\ CA 94709 \\ Akaash Tawade \\ akaasht@berkeley.edu \\ University of California at Berkeley, \\ CA 94709
}

\author{
Manan Khattar \\ mkhattar@berkeley.edu \\ University of California at Berkeley, \\ CA 94709 \\ Adam Bouyamourn \\ adam.bouyamourn@berkeley.edu \\ University of California at Berkeley, \\ CA 94709
}

\begin{abstract}
Alex Devonport
alex_devonport@berkeley.edu University of California at Berkeley,

CA 94709
\end{abstract}

\begin{abstract}
Improving demand response can help optimize renewable energy use and might be possible using current tools in machine learning. We propose an experiment to test the development of Reinforcement Learning (RL) agents to learn to vary a daily grid price signal to optimize behavioral energy shift in office workers. We describe our application of Batch Constrained Q Learning and Soft Actor Critic (SAC) as RL agents and Social Cognitive Theory, LSTM networks, and linear regression as planning models. We report limited success within simulation with SAC and linear regression. Finally, we propose an experiment timeline for consideration.
\end{abstract}

\section{CCS CONCEPTS}

- Hardware $\rightarrow$ Smart grid; Enterprise level and data centers power issues; Impact on the environment; • Applied computing $\rightarrow$ Multicriterion optimization and decision-making.

\section{KEYWORDS}

Demand Response, Transactive Control Learning, Reinforcement Learning, Social Cognitive Theory, Social Energy competitions, Planning models for Reinforcement Learning

ACM Reference Format:

Lucas Spangher, Akash Gokul, Manan Khattar, Joseph Palakapilly, Akaash Tawade, Adam Bouyamourn, Alex Devonport, and Costas Spanos. 2020 Prospective Experiment for Reinforcement Learning on Demand Response in a Social Game Framework. In The Eleventh ACM International Conference

$\overline{{ }^{*} \text { Corresponding author }}$

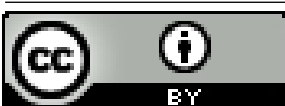

This work is licensed under a Creative Commons Attribution International 4.0 License.

e-Energy'20, June 22-26, 2020, Virtual Event, Australia

(C) 2020 Copyright held by the owner/author(s).

ACM ISBN 978-1-4503-8009-6/20/06.

https://doi.org/10.1145/3396851.3402365

\author{
Costas Spanos \\ spanos@berkeley.edu \\ University of California at Berkeley, \\ CA 94709
}

on Future Energy Systems (e-Energy'20), fune 22-26, 2020, Virtual Event, Australia. ACM, New York, NY, USA, 7 pages. https://doi.org/10.1145/3396851. 3402365

\section{INTRODUCTION AND BACKGROUND LITERATURE}

\subsection{Challenges in Renewable Energy Integration}

Although the Californian grid (CAISO) of 2020 boasted a high renewables penetration of $30 \%$, there are inefficiencies with the way in which this energy was produced and consumed [1]. Specifically, CAISO reported that $3-4 \%$ of its renewable energy was curtailed due to overproduction in 2019[2]. Curtailment was spread unevenly throughout the year, with certain days in the spring reporting 20 $30 \%$ curtailment. In instances like this, the system-wide generation of energy, (specifically solar) was too large for either the electricity grid to demand or for baseline power to adjust down to. Therefore, CAISO simply shut down transmission lines leading to solar panels. The need for curtailment is a problem that will likely grow nonlinearly with respect to the amount of renewable energy on the grid, especially on the local level where transmission from solar farms to the larger grid may be limited.

\subsection{Energy storage and demand response}

Two solutions to curtailment commonly touted are energy storage and demand-response. Energy storage entails the deferment of the consumption from when energy was generated to when it is needed, and Demand-Response (DR) entails the deferment of energy demand from when it is demanded to when it is most opportune for it to be filled. For example, if an electric vehicle were to be plugged in to a charger, a price signal from the grid could cause a controller to charge the EV during the time period in which energy was the least carbon intense, or cheapest to the consumer. DR is basically costless, as it requires no infrastructure, so it is important as a direct solution. However, it can also be a supporting technology for storage. Energy storage provides a measure of security for demands 
that are essential yet faces a seasonality problem: when imagining grids with very high proportion of renewables, batteries need to be sized such that energy stored during a sunny day in the Spring may fill a particularly cloudy day in the Fall [3]. Current storage technologies are not designed with long term storage in mind. DR can help by: (1) alleviating curtailment during high generation months to stretch battery reserves farther, (2) shift seasonal loads (3) alleviate peak loads during the day, to shift demand to other generation sources [4]. Indeed, further understanding the ways in which DR and storage can work together is necessary.

There are other gaps in our understanding of DR. First, it is not clear how much, and what kind of energy demand can be deferred [5]. In the EV example above, we are aware that the EV needs to charge only four hours out of twelve, and so it is an obvious deferable load; however, which other loads could be shown to be deferrable given the right price signal? Can office workers be incentivized to plug in their computers during periods of the day that have cheaper energy, thereby leveraging these batteries as well? Furthermore, which of these deferrable loads, once identified, might be automated so that controls could happen without conscious effort?

\subsection{Social Games and building's energy consumptions}

The share of buildings' energy demands makes up a significant component of US energy demand and is increasing. In residential and commercial buildings, plug loads represent $30 \%$ of total electricity use ([6], [7]). In addition, the quantity of energy used by plugs is increasing more quickly than any other load type in both residential and commercial buildings [8].

To this end, notable work has been performed in creating and administering "Social Games" - for our purposes, defined as competitions around energy use. Social Games tend to comprise of: (1) informing each player of their energy use in a friendly and easy to consume manner and (2) an accompanying competition in which higher energy savings relative to others is rewarded. Many studies have either created Social Games themselves ([9], [10], [11], [12]) or studied existing Social Games ([13], [14]) to draw insight. To this end, the general finding has been that Social Games increase the extent to which an individual is motivated to and able to save energy in their daily functioning within their office or home.

\subsection{Reinforcement Learning for demand response}

Reinforcement Learning is a technique in Machine Learning that trains an agent to optimize actions in an environment. The agent acts within a fairly structured way: it "observes" the environment through a defined state-space, which consists of parameters that the environment can feed to it, and then it "acts" in a way that may modify the local environment; it then observes the effect of its actions through a "reward" that it uses to measure its own success [15]. DR exists within a fairly structured environment itself: energy demands are readily available and predictable, and price signals are typically used to incentivize shifts in energy usage. Therefore, we can easily imagine an agent who can train in producing an appropriate signal for demand response.

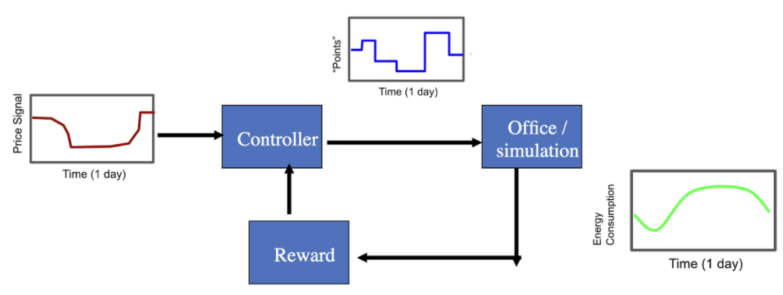

Figure 1: Schematic of the experiment that we will run.

Although DR has been extensively employed and studied within Social Games, we are unaware of a study that attempts to aim a reinforcement learner at DR within a Social Game framework. Therefore, we aim to reproduce a Social Game experiment and quantify the difference in demand response from a variety of RL and RL augmentation techniques.

This paper is an experiment prospective. The rest of the paper is organized as follows: in Section 2, we will first describe the methods in which we hope to implement, describing the RL architectures, planning models, reward specification, and simulation. In section 3, we will show simulated results from the experiment. In Section 4, we will describe the prospective experimental timeline.

\section{METHODS}

As a brief orientation to the experiment, please see Figure 1. Our goal is to design an agent that can translate price signals from the grid to individual workers in an office building to maximize the extend to which they shift their demand. We will propose three different types of agents to implement, and three different planning models to supplement their action. We will then propose a simulation to study the modular components pre-experiment.

\subsection{Reinforcement Learning techniques to consider}

$\mathrm{RL}$ as a general technique came out of the physical simulators and computer gaming - arenas that are data rich in measurements and rewards. They might have access to many steps with millions of iterations to try various trajectories. We are dealing with a different scenario - so-called "human-in-the-loop" learning, where each step in the world is a day and data is very costly to attain.

As such, we need to consider off-policy RL: policies that do not rely solely on interactions with the environment, but with stored data that can be reused, with older data from other experiments, and with intelligent extrapolations. We describe three architectures that we are considering below.

2.1.1 Batch Constrained Q Learning (BCQL). We deal with Q-Learners, which model reward based on (state, action) pairs; i.e. estimating the reward of an agent based on a certain action at a certain state. $Q$ Learning is an model-free method of RL, in that there is no underlying dynamics model. Instead, a prediction model serves to predict the reward that the agent would obtain from each (state, action) 
pair, and then picks the action corresponding to the maximum Qvalue at each step. This is fundamental when you do not have the transition probabilities (i.e. you don't have an underlying dynamics model of the situation.)

Batch-constrained algorithms are ones which straddle policy methods and behavioral cloning, as they take some data in to mimic yet also extrapolate between points. In BCQL, the goal is to train the policy offline, but constrain the agent's actions to within the data distribution of the batch [16]. It uses Actor, Critic, and Deterministic Policy Gradient (DPG) to guide the learning, collecting pairs of states and actions in a replay buffer. It samples actions from a latent space to train a variational auto-encoder (VAE), meaning it maps state action pairs down to a smaller latent space that it is able to search efficiently with the few actions. For this reason, we hypothesize that BCQL would be good for small sample learning. It uses VAE actions with soft clipped double $Q$ learning to get target values and train the critic. A soft clipped double Q-learning means using two $Q$ networks to estimate the critic function by overweighting the smaller $Q$ value, i.e.

$$
Q_{C}=.75 *\left(\min \left(Q_{1}, Q_{2}\right)\right)+.25 *\left(\max \left(Q_{1}, Q_{2}\right)\right)
$$

Then, we generate and use the actions and the critic to calculate DPG and train the actor. Our BCQL agent emits a 10-dim points vector, each output a discrete integer value between 0 and 10 .

2.1.2 Soft Actor Critic (SAC) V2. Traditional Actor Critic employs a form of $Q$ learning with two different prediction networks. One network, the "Critic", predicts the Q-value of actions that are sampled from the policy distribution, i.e. the "Actor" network.

The SAC is an extension of the traditional Actor Critic to a continuous output space, with some improvements to function in an off-policy way [17]. Now, the actor updates the policy distribution with entropy as a regularizing term, maximizing the reward while taking the most "random" step possible. Using entropy regularization makes the actions sampled along the trajectory cover the most ground possible, essentially exploring the space more effectively. Our SAC V2 emits a 10-dim points vector, each output a continuous value between 0 and 10 .

2.1.3 Deep Recurrent $Q$ Networks (DRQN). Following from the brief introduction of $Q$ networks, we are interested in exploring the potential of a $Q$ network with a recurrence built into the policy. Here, the first post-convolutional fully-connected layer of the $Q$ network is replaced with a recurrent LSTM, which allows the agent to incorporate longer term planning into its functioning [18]. Our DRQN will emit a 10-dim points vector, each output a continuous value between 0 and 10 .

2.1.4 Additional technique: Overtraining. In addition to a more routine hyperparameter search, we would like to explore the effect of "overtraining" on the performance of the agent. Here, we will train the agent for successively more iterations before taking steps in the environment. As each time step is important in the training of our agent, we will use the action that produced the minimum reward in the environment. We will apply this technique to all architectures to improve performance.

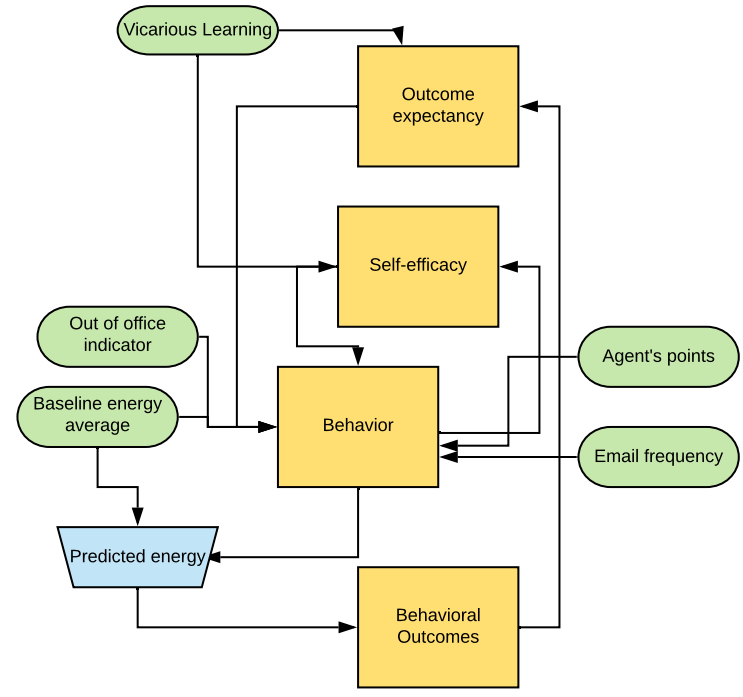

Figure 2: Diagram of the Social Cognitive Model we propose to test. The yellow squares indicate latent states, which are labelled according to their interpretation. The green ovals indicate exogenous inputs, which are also labelled according to their interpretation and are defined in the passage. The blue trapezoid indicates the output, which is a prediction of the energy use that a person will have.

\subsection{Data augmentation and planning: how to improve RL data efficiency}

Although the algorithms that we describe above are important advances in RL for efficient data, we still are concerned that a lack of steps within the environment will hinder any algorithm from effectively learning. We propose a way to address this: planning.

A planning model is one that will be exogenous to the RL agent, which will be focused only on predicting the resulting energy demands given the points that the agent serves. Constructing a good model for human behavior in the office could provide a way for the algorithm to explore more than it could by interfacing with just the world. To this end, we propose three different planning models:

2.2.1 Social Cognitive Theory System. The literature of Social Cognitive Theory proposes the use of linear systems for prediction and control. These systems are highly structured, consisting of latent states and exogenous inputs that relate to each other in defined ways. The extreme level of user specified definition allows us to label latent states based on their exogenous inputs, and suggest some level of interpretability. This is, of course, desired in a model.

We propose to create the SCT Model shown in Figure 2.

In this, there are four latent states:

- Outcome expectancy, i.e. the amount of belief that a player has in the reward that they will get.

- Self-efficacy, i.e. the extent to which a player believes that they have the ability to effect the reward that they get. 
- Behavior, i.e. the extent to which a player reacts to the intervention

- Behavioral outcomes, i.e. the resulting evaluation that a player makes of their behavior and how it matches the outcome.

Furthermore, there are several exogenous variables. We introduce them here but use them throughout the planning models:

- Vicarious learning, i.e. the effect of the actions of others around the player. This is defined per timestep $t$ as the sum of the energy use of people at the player's workstation: $\left(\frac{\sum_{p \in \mathcal{W}} E}{\sum_{p \in \mathcal{W}} 1}\right)$ where $\mathcal{W}$ is the player's workstation.

- Out of Office indicator, i.e. a 1 if the player is in the office or 0 if the player is out of the office. This is sensed through a WiFi-enabled occupancy detection mechanism detailed in [19].

- Baseline Energy, i.e. the average energy consumption in the past, defined per hour $h \in$ day of week $d \in\{$ Mon, Tues, Wed, Thurs, Fri $\}$ as the average of three of the same $h \in d$ of the three week period before the Social Game started.

- Email frequency, i.e. the time since an email was sent reminding the players of the points that they are to play with throughout the day

- Points, i.e. the intervention from the agent.

As the four latent states comprise a 4-tuple $\vec{z}$ and the exogenous inputs comprise a 5-tuple $\vec{u}$, then with transition matrices whose sparsity is implied in Figure 2, $A \in \mathbb{R}^{4 \times 4}$ and $B \in \mathbb{R}^{5 \times 4}$, the following transition is realized:

$$
\vec{z}_{t+1}=A \vec{z}+B \vec{u}
$$

We will explain our attempts to fit data to this in the results.

2.2.2 LSTM Recurrent Neural Networks. The SCT may be seen as a way to explicate structure in exogenous variables by combining subsets of them into different explanatory variables. A more machine learning approach to this would be to allow a neural network to discover the relationships between the variables in a black-box manner.

To this end, we propose training an LSTM network on the aforementioned exogenous variables. We will use basic LSTM formulations in Keras and PyTorch, and compare between them for runtime and accuracy. We will perform a brief neural architecture search, including on the number of stacked LSTM, size of hidden layer, and other hyperparameters, and compare the output based on root mean squared error of prediction on test set, and length of time necessary to train.

2.2.3 Ordinary Least -Squares Regression and Autoregression. In developing these more complex models, we endeavor to have a baseline model to compare against as well. To this end, we propose a brief search into linear regression models. Our linear regressions will consist of the following:

- $y_{t} \sim \vec{u}_{t}$, where $y_{t}$ is the energy at time $t$ and $\vec{u}_{t}$ is the vector of exogenous variables at time $t$.

- $y_{t} \sim y_{t-1}+\ldots y_{t-k}+\vec{u}_{t}$, where $y_{t-1}+\cdots+y_{t-k}$ is a series of autoregressive $y$ 's as determined by analysis of autocorrelation function plot.
- $y_{t} \sim \vec{u}_{t}^{\prime}+p_{t}+p_{t-1} \ldots+p_{t-k}$, where, for notational clarity, $\vec{u}^{\prime}$ is a vector composed of all the exogenous variables except for points $p$, which we draw out to emphasize with a $k^{t h}$ order lag, depending on tests of significance during the first month of experiment system identification.

\subsection{Reward}

All agents will use the same reward calculation. This reward is defined as the difference between the day's total cost of energy and the ideal cost of energy. The ideal cost of energy is obtained using a simple convex optimization. If $\vec{d}$ are the actual demand of energy computed for the day, $\vec{g}$ is the vector of the grid prices for the day, $E$ is the total amount of energy, and $d_{\min }, d_{\max }$ are $5 \%$ and $95 \%$ values of energy observed over the past year, then the ideal demands are calculated with the following optimization:

$$
\begin{aligned}
d^{*}=\min _{d} & d^{T} g \\
\text { s.t. } & \sum_{t=0}^{10} d=E \\
& d_{\text {min }}<d<d_{\text {max }}
\end{aligned}
$$

Then, the reward becomes:

$$
R(d)=\frac{d^{* T} g-d^{T} g}{d^{* T} g}
$$

I.e. taking the difference and scaling by the total ideal cost to normalize the outcome.

\subsection{Simulation design}

In order to test the aforementioned techniques, we will use a simulation that can provide some level of insight into the superiority of some methods over others. We define three deterministic responses to points, listed below. We populate our office with an equal proportion of "people" who exhibit each of the three response types.

2.4.1 Linear Response. Here, we define a very simple person who decreases their energy consumption linearly below a baseline with respect to points given. Therefore, if $b_{t}$ is the baseline energy consumption consumed at time $t$ and $p_{t}$ are the points given, the energy demand $d$ is

$$
d_{t}=b_{t}-p_{t}
$$

This is clipped at $d_{\min }$ and $d_{\max }$ as defined in Section 2.3.

2.4.2 Sinusoidal Response. Here, we define a person who decreases their energy consumption sinusoidally below a baseline with respect to points given. This models a person who might have a low response trigger to the ends of the range of the points. Therefore, given the symbolic representation earlier stated, the equation becomes:

$$
d_{t}=b_{t}-\sin \left(p_{t}\right)
$$

This is clipped at $d_{\min }$ and $d_{\max }$. 


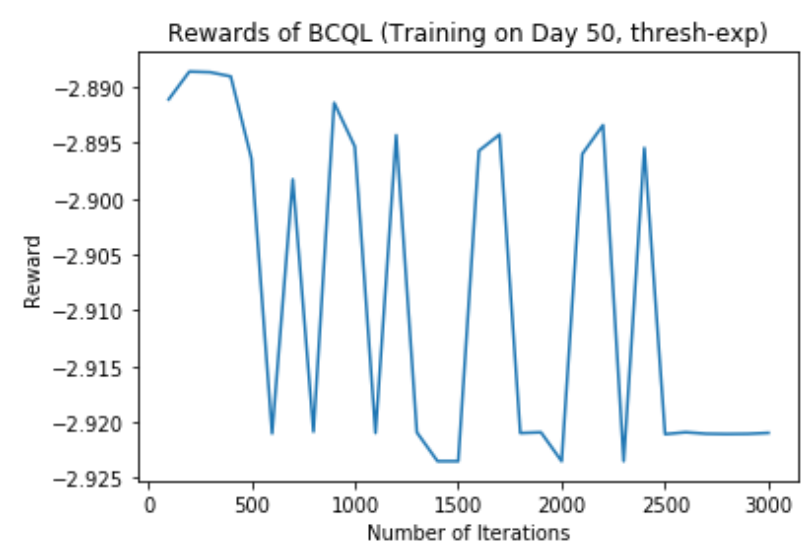

Figure 3: Learning rate of the $\mathrm{BCQL}$ algorithm, trained over 3000 iterations.

2.4.3 Threshold Exponential Response. Here, we define a person who decreases their energy consumption below a baseline exponentially with respect to points given if it passes the threshold of the mean of the points. This is meant to simulate someone who is jolted into a more extreme response when points are large. Therefore, the energy demand $d$ is

$$
d_{t}= \begin{cases}b_{t} & p \leq \bar{p} \\ b_{t}-\exp p_{t} & p>\bar{p}\end{cases}
$$

\section{SIMULATED RESULTS}

Once the agents and planning models were developed, they were tested in the simulation with varying deterministic responses. The agents were allowed to serve up actions and observe the corresponding behavior of the agents, and from there we evaluated how well each of them learned.

\subsection{RL Architectures}

In the RL architecture front, we report limited success with BCQL. Trained over a thirty day period, we see limited evidence that BCQL can learn, and it seems to exhibit little exploration. Please see Figure 3.

We speculate that BCQL's poor behavior is due to it being a fully off-policy algorithm. BCQL can take in data from a series of actions that occurred outside the policy, but the amount of the data required is large. As a result, the model was not able to cover its embedded action space very well. (We confirmed this with a plot of the actions that the agent suggests over 30 days, not plotted here, which reveals almost no deviation beyond a single action sequence.)

However, SAC V2 appears to be performing better. We report that it generally appears to learn across the three deterministic responses. Please see Fig 4.

We have not yet been able to implement DQRN.

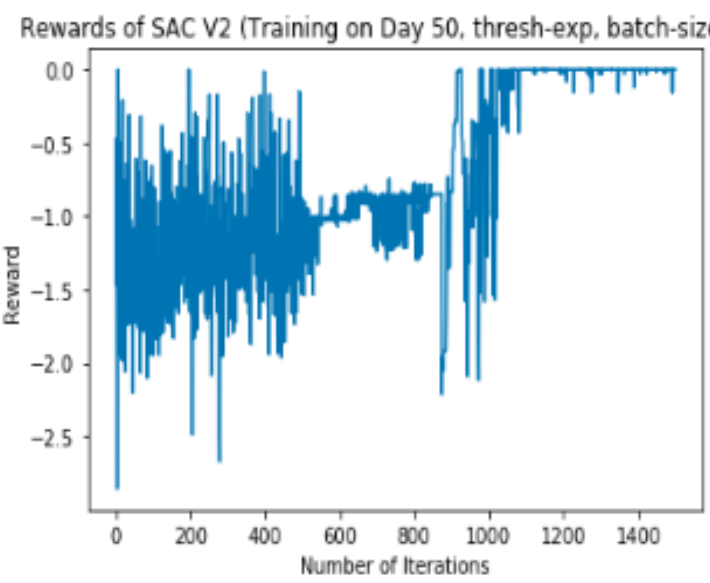

Figure 4: One example run of SAC V2 with threshold exponential response.

\subsection{Overtraining results}

We believe that overtraining holds some promise in helping the policies perform better. We started to anecdotally see improvements to certain models following the implementation of more overtraining, and so we sought to formalize this on a better performing model. We present a summary of multiple training runs presented in figure 5. In this plot, the number of overtraining iterations is varied during a trainig run over 30 days. The sum of rewards across 30 daqys is plotted on the $y$-axis, allowing us to compare the effects of different iteration numbers over many runs. The experiment seems to indicate that the region of 20 to 40 overtraining runs helps to produce a uniformly more positive reward in SAC on the threshold exponential response.

\subsection{Planning models}

3.3.1 SCT Model. Unfortunately, our best efforts at solving the SCT model through optimization failed to produce a method that would help the matrices converge for more than three days worth of energy data - a necessary condition given the length of the experiment. We were able to achieve convergence for one days worth of energy data using the following iterative process:

(1) Initialize A, B, and $\mathrm{z}$ with noise.

(2) For 50 times, do:

(a) With $A, B$ fixed, compute $\vec{z}: z_{t+1}=A z_{t}+B u_{t}$

(b) With $\vec{z}_{\text {old }}$ fixed, and daily demand and daily baseline energy, i.e., $\vec{d}, \vec{b}$, and the third latent state estimate $\vec{z}_{3}=$ $\left(A z_{t}+B u_{t}\right)_{3}$ given, optimize unconstrained for $A, B$ :

$$
A^{*}, B^{*}=\min _{A, B} \sum_{t}\left(\left(d_{t}-b_{t}\right)+\left(A z_{t}+B u_{t}\right)_{3}\right)^{2}
$$

Where the subscript 3 refers to the third element of the vector ensuing from $\left(A z_{t}+B u_{t}\right)$. Being that we were able to get estimates for a single day's transition matrices, we report this nonetheless as 


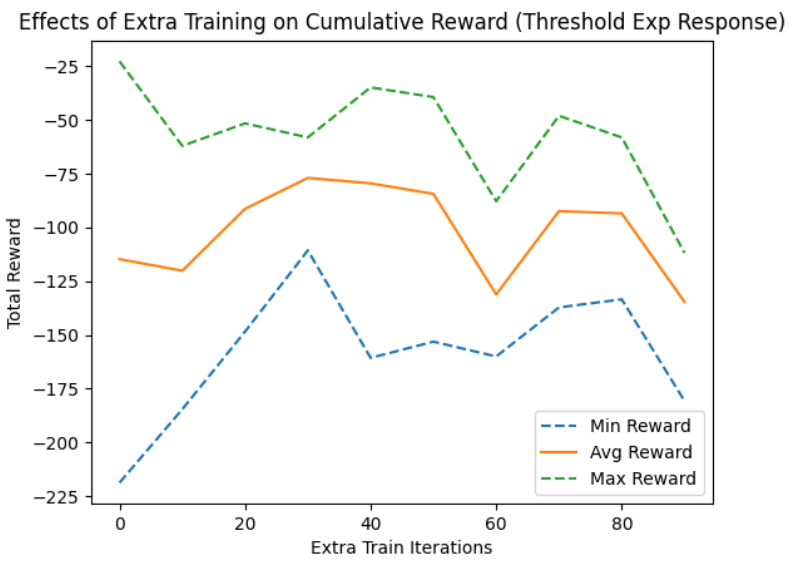

Figure 5: A summary of ten runs of the SAC V2 in an overtraining example. Here, we plot the middle green line as an average and the dotted lines as the minimum and maximum rewards noted in the runs.

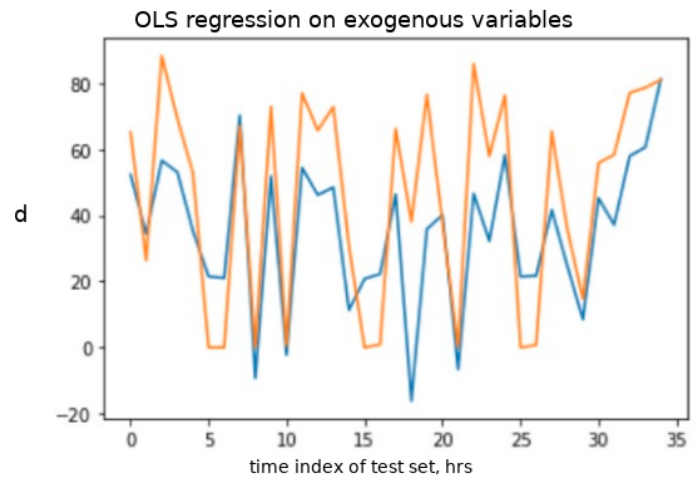

Figure 6: Prediction (blue) with actual (orange) for test set of energy demand.

we believe this bi-layer optimization method has value in solving a system with multiple dependent latent states.

3.3.2 LSTM. We currently do not have LSTM results to report.

3.3.3 Linear regression. We have results from linear regressions to serve as a baseline above which to improve. Here, our best model appears to be $y_{t} \sim b_{t}+p_{t}$, (please see Figure 6) and inclusion of an autoregressive term seems to strictly worsen the predictions. We believe that this is because predicting a longer horizon propogated uncertainty through the end of the prediction.

\section{PROPOSED EXPERIMENTAL TIMELINE}

We observe two experimental units for a period of five months, from August to December. We are interested in estimating the causal effects of two distinct reinforcement learning architectures, $R L_{j}$, for $j \in\{1,2\}$, in addition to the causal effect of combining reinforcement learning with behavioural feedback from the Social Cognitive Model.

We estimate seasonality effects at period $t\left(\delta_{t}\right)$ and improvements in learning due to the accumulation of observations $\left(\Omega_{t}\right)$, by taking the average difference between performance across all conditions at time $t$ and $t_{-1}$. We also estimate the effect of incorporating parameters from the social cognitive model by comparing observations within a single $R L$ architecture, controlling for seasonality. Finally, we causally identify the effect of each $R L_{j}$, which is the difference between the score for $R L_{j}$ versus $R L_{-} j$, and the difference in scores between $R L_{j}$ and $R L_{j}$, conditional on incorporating the social cognitive model, controlling for seasonality in each case.

\begin{tabular}{|c|c|c|c|}
\hline Month & Group 1 & Group 2 & Control \\
\hline July & \multicolumn{3}{|c|}{- System ID - } \\
\hline August & $R L_{2}$ & $R L_{1}+$ plan & \\
\hline September & $R L_{1}$ & $R L_{2}+$ plan & \\
\hline
\end{tabular}

Table 1: Experimental Timeline in which we compare two different RL architectures and the effect of a planning model.

We then use later experimental periods to train the model on smaller subsets of our experimental subjects: smaller groups and then at the individual scale.

\section{CONCLUSION}

We have proposed an experiment to evaluate the potential of using an artifically intelligent agent to improve a grid price signal for demand response. We proposed various reinforcement learning agents and planning models to fulfill this task, and tested them in simulation to determine their efficacy pre-experiment.

\section{ACKNOWLEDGEMENTS}

We would like to acknowledge the generous support of the Singapore-Berkeley Building Efficiency and Sustainability in the Tropics (SinBerBEST) program, which is funded by the National Research Foundation, Prime Minister's Office, Singapore. In addition, we would like to especially thank and acknowledge Ioannis Konstantakopolous [9] for designing the framework of the Social Game.

\section{REFERENCES}

[1] Shucheng Liu. Integrating high penetration renewable energy into the caiso market, 2016.

[2] Mark Specht. Renewable energy curtailment 101. Blog of the Union of Concerned Scientists, 2019.

[3] John P Barton and David G Infield. Energy storage and its use with intermittent renewable energy. IEEE transactions on energy conversion, 19(2):441-448, 2004.

[4] Mohamed H Albadi and Ehab F El-Saadany. A summary of demand response in electricity markets. Electric power systems research, 78(11):1989-1996, 2008.

[5] Josue Campos do Prado and Wei Qiao. A stochastic decision-making model for an electricity retailer with intermittent renewable energy and short-term demand response. IEEE Transactions on Smart Grid, 10(3):2581-2592, 2018.

[6] S Lanzisera, S Dawson-Haggerty, H. Y. I. Cheung, J Taneja, D Culler, and R Brown. Methods for detailed energy data collection of miscellaneous and electronic loads in a commercial office building. Building and Environment, 65:170-177, 2013.

[7] R. S. Srinivasan, J Lakshmanan, E Santosa, and D Srivastav. Plug load densities for energy analysis: K-12 schools,. Energy and Buildings, 43:3289 - 3294, 2011.

[8] O Comstock and K Jarzomski. Consumption and saturation trends of residential miscellaneous end-use loads. ACEEE Summer Study on Energy Efficiency in Buildings, Pacific Grove, CA, USA, 2012 
[9] I. C. Konstantakopoulos, L. J. Ratliff, M. Jin, and C. J. Spanos. Leveraging correlations in utility learning. In 2017 American Control Conference (ACC), pages 5249-5256, May 2017

[10] L. J. Ratliff, M. Jin, I. C. Konstantakopoulos, C. Spanos, and S. S. Sastry. Social game for building energy efficiency: Incentive design. In 2014 52nd Annual Allerton Conference on Communication, Control, and Computing (Allerton), pages 1011-1018, Sep. 2014

[11] T.G. Papaioannou, N. Dimitriou, K. Vasilakis, A. Schoofs, M. Nikiforakis, F. Pursche, N. Deliyski, A. Taha, D. Kotsopoulos, C. Bardaki, S. Kotsilitis, and A. Garbi. An iot-based gamified approach for reducing occupants' energy wastage in public buildings. Sensors (Switzerland), 18(2), 2018.

[12] T.G. Papaioannou and G.D. Stamoulis. Teaming and competition for demand-side management in office buildings. volume 2018-January, pages 332-337, 2018

[13] Ben Cowley, Jose Luiz Moutinho, Chris Bateman, and Alvaro Oliveira. Learning principles and interaction design for "green my place": A massively multiplayer serious game. Entertainment Computing, 2(2):103 - 113, 2011. Serious Games
Development and Applications.

[14] Ian Ayres, Sophie Raseman, and Alice Shih. Evidence from Two Large Field Experiments that Peer Comparison Feedback Can Reduce Residential Energy Usage. The fournal of Law, Economics, and Organization, 29(5):992-1022, 082012

[15] Richard S Sutton and Andrew G Barto. Reinforcement learning: An introduction. MIT press, 2018

[16] Hoang M Le, Cameron Voloshin, and Yisong Yue. Batch policy learning under constraints. arXiv preprint arXiv:1903.08738, 2019.

[17] Tuomas Haarnoja, Aurick Zhou, Pieter Abbeel, and Sergey Levine. Soft actorcritic: Off-policy maximum entropy deep reinforcement learning with a stochastic actor. arXiv preprint arXiv:1801.01290, 2018.

[18] Matthew Hausknecht and Peter Stone. Deep recurrent q-learning for partially observable mdps. In 2015 AAAI Fall Symposium Series, 2015.

[19] Han Zou, Hari Prasanna Das, Jianfei Yang, Yuxun Zhou, and Costas Spanos. Machine learning empowered occupancy sensing for smart buildings. 